\title{
Diagnostic Performance of PET Versus SPECT Myocardial Perfusion Imaging in Patients with Smaller Left Ventricles: A Substudy of the ${ }^{18}$ F-Flurpiridaz Phase III Clinical Trial
}

\author{
René R. Sevag Packard ${ }^{1-3}$, Joel L. Lazewatsky ${ }^{4}$, Cesare Orlandi ${ }^{4}$, and Jamshid Maddahi ${ }^{1,5}$ \\ ${ }^{I}$ Division of Cardiology, Department of Medicine, David Geffen School of Medicine, UCLA, Los Angeles, California, ${ }^{2}$ Ronald Reagan \\ UCLA Medical Center, Los Angeles, California; ${ }^{3}$ Veterans Affairs West LA Medical Center, Los Angeles, California; ${ }^{4}$ Lantheus \\ Medical Imaging, North Billerica, Massachusetts; and ${ }^{5}$ Nuclear Medicine Clinic, Department of Molecular and Medical Pharmacology, \\ David Geffen School of Medicine, UCLA, Los Angeles, California
}

The performance of SPECT myocardial perfusion imaging (MPI) may deteriorate in smaller hearts, primarily because of the lower resolution of conventional Anger cameras. ${ }^{18} \mathrm{~F}$-flurpiridaz is a novel PET MPI agent with superior image and defect resolution. We sought to determine the diagnostic performance of ${ }^{99 \mathrm{~m}}$ Tc-labeled SPECT MPI compared with ${ }^{18} \mathrm{~F}$-flurpiridaz PET MPI according to left ventricle (LV) size. Methods: We conducted a substudy of the phase III clinical trial of flurpiridaz $(n=750)$ and stratified diagnostic performance according to the median PET LV end-diastolic volume (LVEDV), with smaller LVs defined as having an LVEDV of less than $113 \mathrm{~mL}(n=369)$ and larger LVs defined as having an LVEDV of at least $113 \mathrm{~mL}(n=381)$. Images were interpreted by the majority rule of 3 independent masked readers. The reference standard was quantitative invasive angiography, with at least $50 \%$ stenosis in at least 1 coronary artery considered significant. Results: SPECT performance decreased significantly from an area under the curve (AUC) of 0.75 in larger LVs to 0.67 in smaller LVs $(P=0.03)$, whereas PET performance was similar in larger and smaller LVs (AUC, 0.79 vs. $0.77, P=0.49$ ). Accordingly, in smaller LVs, PET had a higher AUC (0.77) than the SPECT AUC $(0.67)(P<$ $0.0001)$, a phenomenon driven by female patients $(P<0.0001)$. In smaller LVs, there was a degradation of SPECT sensitivity that was highly significant $(P<0.001)$, whereas there was no significant change in PET sensitivity according to LV size $(P=0.07)$. Overall, PET had significantly higher sensitivity than SPECT in both smaller LVs (67\% vs. $43 \%, P<0.001)$ and larger LVs $(76 \%$ vs. $61 \%, P<0.001)$. The specificities of PET and SPECT were similar in larger LVs $(76 \%$ vs. $83 \%, P=0.11)$. Although SPECT specificity improved in smaller compared with larger LVs (90\% vs. $83 \%, P=0.03)$, the PET specificity did not change with LV size ( $76 \%$ vs. $76 \%, P=0.9)$. Conclusion: The diagnostic performance of ${ }^{18} \mathrm{~F}$-flurpiridaz PET MPI is not affected by LV size and is superior to SPECT MPI in patients with smaller LVs, highlighting the importance of appropriate test selection in these patients.

Key Words: diagnostic performance; left ventricle size; SPECT MPI; PET MPI; flurpiridaz

J Nucl Med 2021; 62:849-854

DOI: 10.2967/jnumed.120.252007

Received Jun 30, 2020; revision accepted Nov. 4, 2020.

For correspondence or reprints, contact René R. Sevag Packard (rpackard@mednet.ucla.edu).

Published online Nov. 27, 2020.

COPYRIGHT (C) 2021 by the Society of Nuclear Medicine and Molecular Imaging.
$\mathbf{L}$ height, and sex, as well as across ethnic groups, such as between Caucasian and Asian populations (1-5). An ideal noninvasive imaging test should have preserved diagnostic value irrespective of left ventricle (LV) size. However, using receiver-operating-characteristic (ROC) analyses, the diagnostic performance of SPECT myocardial perfusion imaging (MPI) for detection of coronary artery disease (CAD) has been reported to be lower in smaller hearts ( 1 ) in the setting of suboptimal spatial resolution and low myocardial extraction fractions of technetium-based radiopharmaceuticals, leading to limitations in defect resolution (6). The suboptimal spatial resolution of SPECT is further demonstrated by spuriously high determinations of ejection fraction $(7,8)$.

PET MPI is increasingly being used in clinical practice $(6$, 9-11) and further allows for the accurate quantitation of myocardial blood flow (12). ${ }^{18} \mathrm{~F}$-flurpiridaz is a novel PET MPI agent that has undergone phase II (13) and III (14) trial evaluation. ${ }^{18} \mathrm{~F}$-flurpiridaz has a high image resolution due to the short positron range of ${ }^{18} \mathrm{~F}$, the improved spatial resolution of PET over SPECT, routine attenuation correction for PET, and a superior defect resolution due to the elevated myocardial extraction fraction of this radiopharmaceutical $(9,11,12)$.

Large metaanalyses suggest superior diagnostic performance in global populations of PET compared with SPECT MPI $(15,16)$, in addition to significantly reduced radiation exposure (17). However, in patients with smaller hearts being tested for CAD, the nuclear cardiology modality with the highest diagnostic yield is unknown. To help fill this gap in knowledge and clinical care, we sought to systematically compare the diagnostic performance of ${ }^{99} \mathrm{~m}$ Tc-labeled SPECT MPI and ${ }^{18}$ F-flurpiridaz PET obtained sequentially in the same 750 patients enrolled in the phase III trial of flurpiridaz, with results stratified according to LV size.

\section{MATERIALS AND METHODS}

\section{Patient Population}

In the present study, all 755 patients from the first phase III trial of ${ }^{18}$ F-flurpiridaz PET were evaluated (14). Five studies with corrupt gated data were excluded (final $n=750$ ). The trial design was a prospective, open-label study comparing the performance of ${ }^{99 \mathrm{~m}}$ Tc-labeled SPECT MPI versus ${ }^{18} \mathrm{~F}$-flurpiridaz PET in patients referred for invasive coronary angiography (ICA) (ClinicalTrials.gov identifier NCT01347710). The study was approved by local institutional review boards, and all patients provided written informed consent. Briefly, 
eligible patients had known or suspected CAD and either had SPECT and PET MPI done before ICA or, alternatively, underwent ICA without intervention, after which both nuclear imaging tests were obtained. Both MPIs were performed within $60 \mathrm{~d}$ of ICA. Significant exclusion criteria included myocardial infarction or unstable angina within 6 mo, percutaneous coronary intervention within $6 \mathrm{mo}$, a history of coronary artery bypass grafting, New York Heart Association class III/IV heart failure, nonischemic cardiomyopathy, symptomatic valvular disease, significant congenital heart disease, a history of heart transplantation, or a transient ischemic attack or cerebrovascular accident within 3 mo.

\section{Study Protocol}

The mode of stress was either exercise ( $n=221,29 \%)$ or pharmacologic $(n=534,71 \%)$, with the identical stress modality used in both the SPECT study and the PET study of each patient. SPECT MPI was performed without attenuation correction, as per common clinical practice. Flurpiridaz doses were prespecified as $92.5-111 \mathrm{MBq}$ (2.5-3.0 mCi) for rest, 333-351.5 MBq (9.0-9.5 mCi) for exercise stress ( $\geq 60 \mathrm{~min}$ rest-stress interval), and $222-240.5 \mathrm{MBq}(6.0-6.5$ $\mathrm{mCi}$ ) for pharmacologic stress with regadenoson, adenosine, or dipyridamole ( $\geq 30$ min rest-stress interval) (16). Raw data were submitted to a central laboratory (BioClinica, Inc.) for quality control and processing for masked interpretation by 3 independent expert readers, coordinated and conducted by the core laboratory. The reference standard was quantitative ICA assessed by the clinical trial core lab in a masked manner (PERFUSE Core Laboratory), with significant disease defined as at least $50 \%$ stenosis in at least 1 coronary artery.

\section{Volumes}

LV end-diastolic volumes (LVEDVs) were determined from gated rest ${ }^{18} \mathrm{~F}$-flurpiridaz PET images using Corridor4DM software. Studies were divided into subgroups according to the median LVEDV $(113 \mathrm{~mL})$ or by quartiles. Smaller ventricles were defined as an LVEDV of less than $113 \mathrm{~mL}(n=369$ patients), and larger ventricles were defined as an LVEDV of at least $113 \mathrm{~mL}(n=381$ patients). The diagnostic performances of PET and SPECT, defined as ROC areas under the curve (AUCs), were then compared according to LV size.

\section{MPI Analyses}

The primary efficacy read for MPI status was the overall qualitative diagnosis determination based on each independent read using perfusion and gated image data only. Reads were dichotomized as MPI-negative (normal) or MPI-positive for each patient. The majority rule was used for sensitivity and specificity, where at least 2 readers had the same interpretation. The semiquantitative read was conducted using the 17-segment LV model with a multipoint grading scale $(0-4)$. Summed stress scores were used to derive ROC curves for SPECT and PET. ROC analyses of AUCs were performed using the median value of the resulting summed stress score for each patient and imaging modality. ROC curves are presented as AUC and Wald $95 \%$ CIs.

\section{Statistical Analyses}

Patient characteristics were compared using the $\chi^{2}$ test for categoric variables and the $t$ test for continuous variables. A Spearman rank correlation was performed to determine the relationship of LVEDV with baseline characteristics. Sensitivity and specificity comparisons were performed using the 2-sided McNemar test at an $\alpha$-value of 0.05 . The differences in ROC AUC, with associated $P$ value, were determined by $\chi^{2}$ analyses. A $P$ value of less than 0.05 was considered significant. SAS, version 9.3, was used for analyses.

\section{RESULTS}

\section{Patient Characteristics}

The distribution of patient characteristics according to LV size categories is shown in Table 1. Patients were divided according to the median LVEDV, leading to 369 patients in the smaller-LV group (LVEDV $<113 \mathrm{~mL}$ ) and 381 patients in the larger-LV group (LVEDV $\geq 113 \mathrm{~mL}$ ). Other than age and tobacco-use history, traditional cardiovascular risk factors were distributed similarly between groups. Patients with smaller LVs had smaller height, weight, body mass index (BMI), and body surface area (BSA).

\section{LVEDV Distribution and Correlation with Patient Parameters}

The distribution of LVEDV and corresponding patient numbers is illustrated in Figure 1A. The median LVEDV was $113 \mathrm{~mL}$, with an interquartile range of $92-143 \mathrm{~mL}$ and a minimal-to-maximal value spread of 41-423 mL. The correlation of LVEDV with pertinent patient parameters is further presented in Table 2. The lowest correlation was with BMI $(r=0.24)$; correlations were significantly higher with weight $(r=0.51)$, height $(r=0.54)$, and BSA ( $r=0.59$ ) (all with $P<0.0001)$. The parameter values correlating with the median LVEDV $(113 \mathrm{~mL})$ were $1.73 \mathrm{~m}$ for height, 89.4 $\mathrm{kg}$ for weight, $30 \mathrm{~kg} / \mathrm{m}^{2}$ for BMI, and $2.03 \mathrm{~m}^{2}$ for BSA. Of the study subjects, $332(44 \%)$ had a BSA of $2.0 \mathrm{~m}^{2}$ or less. The correlation plot between BSA and LVEDV is illustrated in Figure 1B.

\section{Diagnostic Performance of SPECT Versus PET MPI According to LV Size}

The ROC curves for SPECT versus PET in smaller and larger LVs are presented in Figure 2. In smaller LVs (Fig. 2A), PET had a higher AUC $(0.77 ; 95 \%$ CI, 0.72-0.82) than did SPECT $(0.67$; 95\% CI, 0.62-0.72) ( $P<0.0001)$. In larger LVs (Fig. 2B), PET similarly had a higher AUC $(0.79 ; 95 \%$ CI, 0.75-0.84) than did SPECT $(0.75 ; 95 \%$ CI, $0.70-0.79)$; however this difference was borderline-insignificant $(P=0.06)$. We additionally explored the diagnostic performance of SPECT versus SPECT and PET versus PET according to LV size (Supplemental Fig. 1; supplemental materials are available at http://jnm.snmjournals.org). SPECT performance decreased significantly from larger to smaller LVs ( $P=0.03$ ) (Supplemental Fig. 1A). On the other hand, PET had a similar performance in larger and smaller LVs $(P=0.49)$ (Supplemental Fig. 1B). Our findings indicate that although there was a significant decline in the diagnostic performance of SPECT in smaller ventricles, the diagnostic performance of PET did not change significantly with LV size. When PET correctly identified perfusion defects (true-positives) and SPECT did not (falsenegatives), the prevalence of multivessel CAD was similar between smaller $(n=11,30 \%)$ and larger $(n=14,36 \%)$ LVs $(P=$ 0.63) (Supplemental Table 1). Taken together, these results demonstrate the superior diagnostic performance of PET over SPECT, and this superiority was most pronounced in smaller ventricles.

We subsequently stratified the diagnostic performance of SPECT versus PET by quartile groupings of LV size (Fig. 3). In patients with very small LVs (Fig. 3A, quartile 1: LVEDVs of $\geq 41 \mathrm{~mL}$ to $<92 \mathrm{~mL})$, the PET AUC $(0.74 ; 95 \% \mathrm{CI}, 0.66-0.81)$ was superior to the SPECT AUC $(0.64 ; 95 \% \mathrm{CI}, 0.57-0.71)(P<$ 0.05). Similarly, in patients with LVs in quartile 2 (Fig. $3 \mathrm{~B}$, LVEDVs of $\geq 92 \mathrm{~mL}$ to $<113 \mathrm{~mL})$, the PET AUC $(0.79 ; 95 \% \mathrm{CI}$, $0.73-0.86)$ was greater than the SPECT AUC $(0.70 ; 95 \% \mathrm{CI}$, 0.63-0.77) $(P<0.01)$. Even in patients whose LVs were larger than the median (Fig. 3C, quartile 3: LVEDVs of $\geq 113 \mathrm{~mL}$ to $<143 \mathrm{~mL}$ ), the diagnostic performance of PET (AUC, $0.75 ; 95 \%$ 
TABLE 1

Patient Characteristics According to LV Size

\begin{tabular}{lccc}
\hline Characteristic & Smaller LV $(n=369)$ & Larger LV $(n=381)$ & $P$ \\
\hline Age $(\mathrm{y})$ & $64.2 \pm 9.7$ & $60.6 \pm 9.0$ & $<0.0001$ \\
Sex, male & $185(50)$ & $335(88)$ & $<0.0001$ \\
Hypertension & $305(82)$ & $315(83)$ & 0.80 \\
\hline Dyslipidemia & $323(87)$ & $328(86)$ & 0.77 \\
\hline Diabetes mellitus & $124(33)$ & $134(35)$ & 0.60 \\
\hline Tobacco use history & $196(53)$ & $255(67)$ & $<0.0001$ \\
Family history of CAD & $230(62)$ & $212(56)$ & 0.09 \\
\hline Height $(\mathrm{m})$ & $1.68 \pm 0.1$ & $1.76 \pm 0.09$ & $<0.0001$ \\
Weight $(\mathrm{kg})$ & $83.5 \pm 17.7$ & $99.6 \pm 19.0$ & $<0.0001$ \\
\hline BMI $\left(\mathrm{kg} / \mathrm{m}^{2}\right)$ & $29.8 \pm 6.2$ & $32.3 \pm 6.8$ & $<0.0001$ \\
BSA $\left(\mathrm{m}^{2}\right)$ & $1.9 \pm 0.2$ & $2.2 \pm 0.2$ & $<0.0001$ \\
\hline
\end{tabular}

Data are mean \pm SD or number followed by percentage in parentheses. For BSA calculations, Du Bois formula was used (35), where $\mathrm{BSA}=0.007184 \times$ height $(\mathrm{m})^{0.725} \times$ weight $(\mathrm{kg})^{0.425}$.

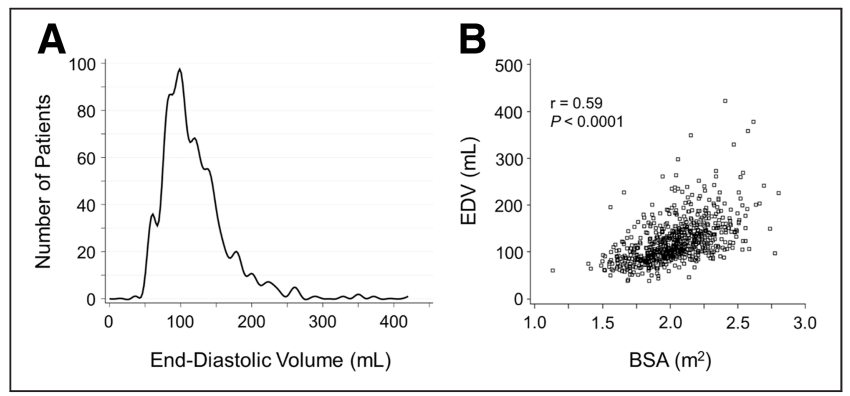

FIGURE 1. Distribution of LVEDVs in trial population (A) and Spearman correlation of BSA with LVEDV (B). EDV = LVEDV.

CI, 0.68-0.81) was superior to that of SPECT (AUC, 0.67; 95\% CI, 0.60-0.74) $(P<0.05)$. Only in patients with the largest ventricles (Fig. 3D, quartile 4: LVEDVs of $\geq 143 \mathrm{~mL}$ to $\leq 423$ $\mathrm{mL}$ ) was the diagnostic performance of PET (AUC, 0.83; 95\% CI, 0.77-0.89) similar to that of SPECT (AUC, 0.82;95\% CI, $0.77-0.88)(P=0.85)$.

The sensitivity and specificity of SPECT and PET based on dichotomous visual reads and stratified according to LV size are presented in Figure 4 . There was a highly significant degradation of SPECT sensitivity in smaller versus larger LVs $(43 \%$ and $61 \%$, respectively, $P<0.001)$. However, there was no significant change in PET sensitivity according to LV size $(67 \%$ in smaller LVs vs. $76 \%$ in larger LVs, $P=0.07$ ). Thus, PET had significantly higher

TABLE 2

Correlations of Patient Parameters with LVEDV

\begin{tabular}{lcc}
\hline Parameter & Spearman $\rho$ & $P$ \\
\hline Height & 0.54 & $<0.0001$ \\
Weight & 0.51 & $<0.0001$ \\
BMI & 0.24 & $<0.0001$ \\
BSA & 0.59 & $<0.0001$ \\
\hline
\end{tabular}

sensitivity than SPECT in both smaller LVs (67\% vs. $43 \%$, respectively, $P<0.001)$ and larger LVs (76\% vs. $61 \%$, respectively, $P<$ 0.001) (Fig. 4A). On the other hand, the specificities of SPECT and PET were similar in larger LVs $(76 \%$ vs. $83 \%$, respectively, $P=$ 0.11 ), and the specificity of SPECT was superior to that of PET in smaller LVs $(90 \%$ vs. $76 \%$, respectively, $P<0.001)$ (Fig. 4B). Of note, this finding was driven by a significant improvement in SPECT specificity in smaller compared with larger LVs (83\% vs. $90 \%$, respectively, $P=0.03)$, whereas PET specificity did not change with LV size ( $76 \%$ vs. $76 \%, P=0.9)$.

We further scrutinized the diagnostic performance of SPECT versus PET in smaller LVs according to sex (Fig. 5). In men (Fig. 5A), PET had an AUC $(0.75 ; 95 \%$ CI, 0.68-0.82) similar to that of SPECT $(0.71 ; 95 \%$ CI, 0.65-0.77) $(P=0.24)$. In women (Fig. 5B), PET had a significantly higher AUC $(0.76 ; 95 \%$ CI, 0.68-0.84) than did SPECT $(0.61 ; 95 \%$ CI, 0.52-0.70) $(P<0.0001)$.

\section{Case Examples of Discrepant MPI Studies}

The pharmacologic stress MPI results of a 66-y-old woman with an LVEDV of $82 \mathrm{~mL}$ are presented in Figure 6. Her SPECT MPI findings (Fig. 6A) were interpreted as definitely normal, whereas her PET MPI findings (Fig. 6B) were deemed definitely abnormal. ICA

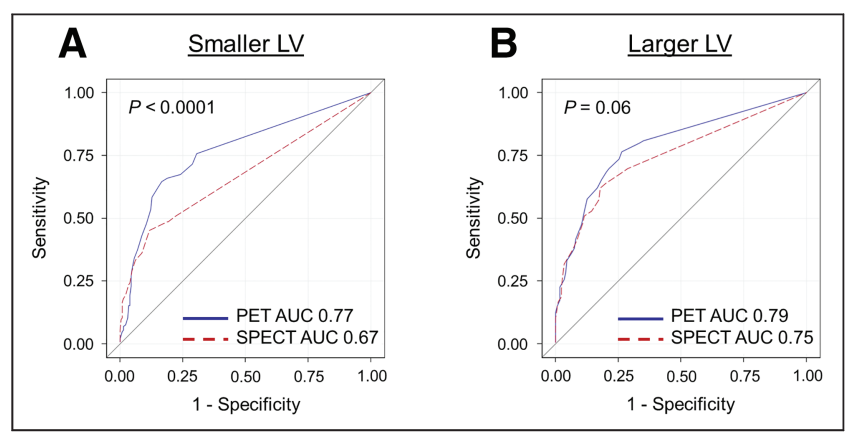

FIGURE 2. Diagnostic performance of SPECT vs. PET according to LV size. Performance of both imaging modalities against each other is compared in smaller (A) and in larger (B) LVs. 

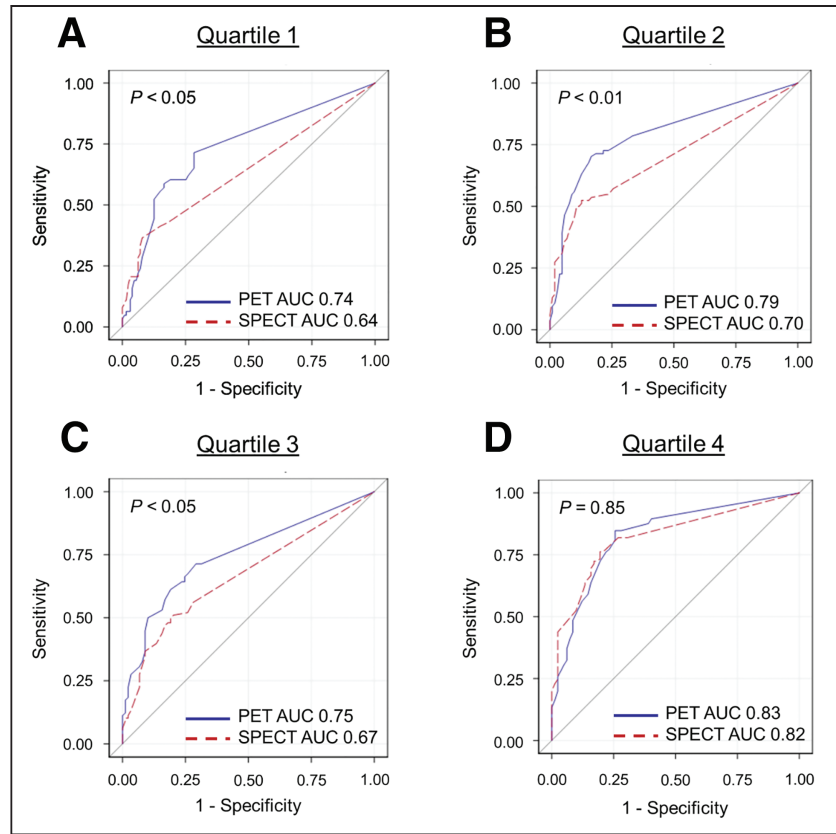

FIGURE 3. Diagnostic performance of SPECT vs. PET according to quartiles of LV size. Performance of both imaging modalities against each other is compared in quartiles $1(A), 2$ (B), $3(C)$, and 4 (D).

demonstrated 2-vessel disease with stenoses of $82 \%$ in the left anterior descending coronary artery, $54 \%$ in the left circumflex coronary artery, and $43 \%$ in the right coronary artery.

We further depict the pharmacologic stress MPI results of a 72y-old man with an LVEDV of $88 \mathrm{~mL}$ (Supplemental Fig. 2). His SPECT MPI results (Supplemental Fig. 2A) were reported as definitely normal, whereas his PET MPI results (Supplemental Fig. 2B) were assessed as definitely abnormal. ICA demonstrated significant $\mathrm{CAD}$, with stenoses of $46 \%$ in the left anterior descending coronary artery, $0 \%$ in the left circumflex coronary artery, and $100 \%$ in the right coronary artery.

\section{DISCUSSION}

At present, societal guidelines and appropriate-use criteria (18, 19) do not favor selecting different cardiac imaging modalities based on LV size to enhance diagnostic performance and optimize patient risk stratification, in part because of a paucity of robust data. An early study by Hansen et al. using ${ }^{201}$ TI SPECT MPI demonstrated that smaller chamber size was associated with a

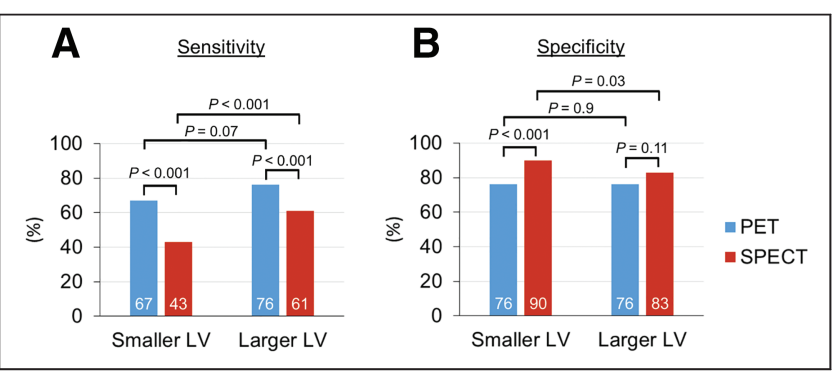

FIGURE 4. Sensitivity and specificity of SPECT and PET in smaller and in larger LVs. Sensitivity (A) and specificity (B) of both imaging modalities are compared with each other in smaller and in larger LVs.
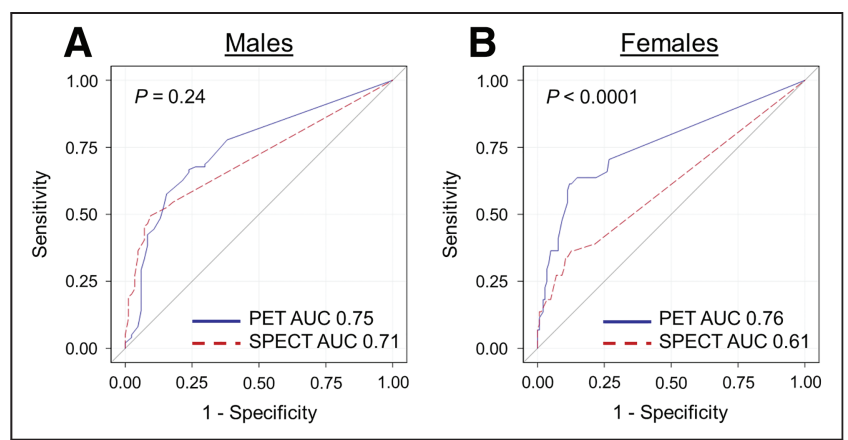

FIGURE 5. Diagnostic performance of SPECT vs. PET in smaller LVs according to sex. Performance of both imaging modalities in smaller LVs is compared against each other in men (A) and in women (B).

significant detrimental effect on diagnostic performance (1). Further, cardiac MRI measures of LV volumes differ significantly according to body size in addition to sex (2). Interestingly, quantitative SPECT MPI studies using commercial software packages in Japanese (3) and Chinese (4) populations demonstrated a loss of diagnostic performance in such patients, a finding that the investigators propose to be driven at least in part by smaller hearts than in Western patients, and independent of sex.

Factors affecting PET image resolution include the positron range, with higher positron emission energies such as that of ${ }^{82} \mathrm{Rb}$ associated with higher positron ranges and image blurring (9). In addition, routine attenuation correction and a reduced prevalence of artifacts further enhance the diagnostic performance, risk stratification, and predictive ability of PET MPI (20). To date, this has been particularly demonstrated in obese patients (21). Indeed, higher image resolution and routine measurement of attenuation correction in PET MPI decrease falsepositive results, particularly in the presence of breast tissue or significant adipose tissue, thereby increasing specificity (11). In addition, higher spatial and contrast resolution, and elevated PET radiopharmaceutical myocardial extraction fraction-particularly with ${ }^{18} \mathrm{~F}$-flurpiridaz (22) - improve the detection of perfusion-abnormalities, thereby decreasing false-negative findings and increasing sensitivity $(10,12,13)$. Furthermore, PET MPI permits identification of cardiac function at peak vasodilatory stress (23) and is ideally suited for absolute myocardial blood flow quantitation $(12,24,25)$, both of which are associated with enhancement of diagnosis and also contribute to prognostic determination. Thus, PET MPI permits integration of perfusion, flow, and cardiac function.

Flotats et al. reported that ${ }^{82} \mathrm{Rb}$-chloride PET MPI offers higher image quality and interpretation confidence than ${ }^{99 \mathrm{~m}} \mathrm{Tc}$-labeled SPECT MPI with or without attenuation correction, obtained in the same patients (26). Similarly, in phase II (13) and III (14) trials, the image quality and diagnostic certainty were found to be better with ${ }^{18} \mathrm{~F}$-flurpiridaz than with ${ }^{99 \mathrm{~m}} \mathrm{Tc}$-labeled SPECT MPI performed in the same patients. Metaanalyses of SPECT versus PET MPI for the detection of CAD with ICA as the reference standard $(15,16)$ have confirmed the superior diagnostic performance of PET in global patient populations. Whether this remains true in patients with smaller ventricles has not been appropriately studied. Bateman et al. compared ${ }^{99 \mathrm{~m}} \mathrm{Tc}$-sestamibi SPECT versus ${ }^{82} \mathrm{Rb}$-chloride PET MPI in separate but matched patient populations, concluding that diagnostic performance was higher in PET 


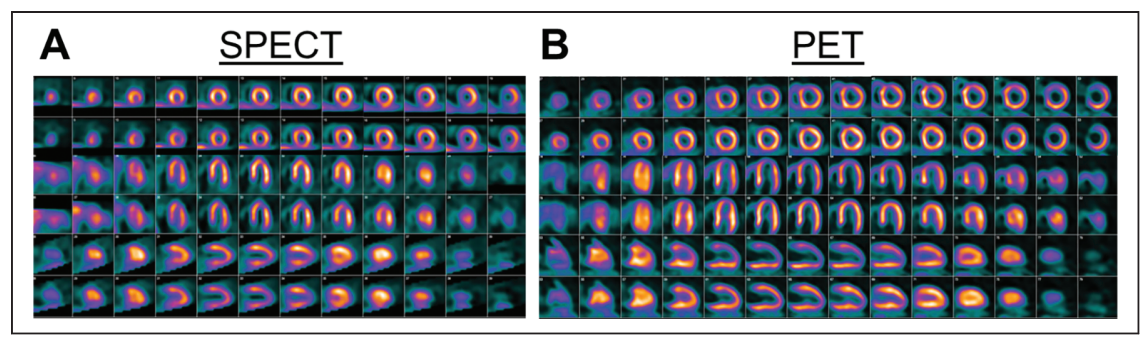

FIGURE 6. Case example: reformatted myocardial perfusion images (stress images above corresponding rest images) of 66-y-old woman with LVEDV of $82 \mathrm{~mL}$. Although SPECT images (A) were interpreted as normal, PET images (B) demonstrated anterior and lateral wall stress-inducible defects, consistent with underlying $82 \%$ stenosis in left anterior descending coronary artery and $54 \%$ stenosis in left circumflex coronary artery.

independent of sex and BMI (27). Their results, however, were not stratified according to LV size.

To the best of our knowledge, no prior large study has looked at the diagnostic performance of SPECT versus PET MPI in smaller versus larger ventricles in a systematic manner and in the same patients, similar to our current study. Our results indicate the superiority of PET over SPECT not only in patients with smaller LVs (defined as being smaller than the study population median) but also in very small LVs (1st-24th percentiles) as well as in somewhat larger LVs (51st-74th percentiles). Indeed, only in patients with the largest LVs (75th-100th percentiles) was the diagnostic performance of SPECT similar to that of PET. Further, we present observations with flurpiridaz that permit both pharmacologic and exercise stress, not routinely performed in clinical practice because of the short half-lives of the currently available PET radiopharmaceuticals (9). Importantly, only $59 \%$ of obese patients with a BMI of at least $30 \mathrm{~kg} / \mathrm{m}^{2}$ in our study had a small LV, suggesting that the benefit from PET in this patient population is largely due to soft-tissue attenuation correction. Furthermore, our study indicates that there is a significant benefit of PET in female patients with smaller LVs, likely driven at least in part by breast and adipose tissue attenuation correction.

Several factors may have influenced the diagnostic performance of SPECT and PET MPI in the phase III clinical trial of flurpiridaz (14). There are no prior clinical trials comparing the performance of SPECT and PET MPI in the same patients before or after ICA. Thus, our results may differ from observations in the published literature $(15,16)$. Overall sensitivity may have been somewhat lower, given the potential safety concerns about delaying coronary revascularization in patients with high-risk CAD determined during ICA or with a very abnormal SPECT MPI result, leading to such patients not being enrolled in the trial. The reference standard was percentage stenosis by ICA, which has a poor relation to the functional significance of CAD by fractional flow reserve (28) or MPI (29), thus adversely affecting both sensitivity and specificity.

Analyses from the first phase III trial of flurpiridaz demonstrated that masked visual analysis of SPECT was skewed toward higher specificity and lower sensitivity (14). This bias is obviated by ROC AUC analyses, which provide an objective assessment of diagnostic performance. A second phase III trial of flurpiridaz is ongoing (ClinicalTrials.gov identifier NCT03354273). In view of the subjectivity of direct comparison of PET versus SPECT MPI, the primary outcome measure will be the diagnostic performance of ${ }^{18} \mathrm{~F}$-flurpiridaz PET MPI in the detection of significant CAD, defined as at least $50 \%$ stenosis. Comparison of the diagnostic performance of PET versus SPECT MPI will be a secondary outcome measure.

Identifying the ideal noninvasive test for the appropriate patient is paramount, as smaller hearts are associated with a lower sensitivity to detect obstructive $\operatorname{CAD}(1,3,4)$. Our current imaging strategies for detection of CAD thus require further refinement. The present body of work supports an expanded role for PET MPI over SPECT MPI in the diagnosis of CAD in patients with smaller LVs. Importantly, our findings further add to the body of work supporting ${ }^{18} \mathrm{~F}$-flurpiridaz as an ideal PET perfusion tracer with superior diagnostic performance $(9,13,14,24,25,30-33)$.

This study had some limitations. Reference ranges for cardiac volumes derived from PET MPI are not well defined. For example, a study comparing 4 different commercial software packages demonstrated that LV volumes derived from gated ${ }^{82} \mathrm{Rb}$-chloride PET MPI varied considerably (34). Future studies will need to establish the correlation of cardiac volumes obtained by ${ }^{18} \mathrm{~F}$-flurpiridaz with the reference standards of cardiac MRI and transthoracic echocardiography and ideally compare results with other available PET radiopharmaceuticals. To reflect routine clinical practice, SPECT MPI was conducted without CT attenuation correction, and this choice may have affected SPECT sensitivity and specificity. Finally, our results need to be tested prospectively in future studies to be appropriately validated and potentially integrated into routine clinical care. To facilitate such studies and assist physicians in the selection of the most appropriate imaging modality, we determined the association of patient characteristics routinely obtained during a clinical visit with LVEDV. Of height, weight, BMI, and BSA, the best correlation was between BSA and LVEDV.

\section{CONCLUSION}

The present study compared the diagnostic performance of SPECT versus PET according to LV size. The diagnostic performance of ${ }^{18} \mathrm{~F}$ flurpiridaz PET MPI for detection of CAD is not significantly affected by LV size. The superior performance of ${ }^{18} \mathrm{~F}$-flurpiridaz PET compared with ${ }^{99 \mathrm{~m}} \mathrm{Tc}$-labeled SPECT is more pronounced in smaller ventricles and is driven by a patient's being female, underscoring the added value of intrinsic attenuation correction in addition to the higher spatial resolution of PET. Future studies should prospectively analyze whether patients with smaller LVs benefit more from PET than from SPECT MPI.

\section{DISCLOSURE}

Funding was provided by NIH 1R43HL123069-01, NIH 2R44HL123069-02, VA Merit BX004558, and Lantheus Medical Imaging. The phase III trial analyzing flurpiridaz MPI was funded by Lantheus Medical Imaging. Joel Lazewatsky is a current, and Cesare Orlandi a former, employee of Lantheus Medical Imaging. Jamshid Maddahi is a scientific advisor to Lantheus Medical Imaging. No other potential conflict of interest relevant to this article was reported.

\section{ACKNOWLEDGMENTS}

We thank Neil Wohlford and Jeffrey Joseph, both at Chiltern, for statistical support. 
KEY POINTS

QUESTION: What is the diagnostic performance of ${ }^{99 \mathrm{~m}} \mathrm{Tc}$-labeled SPECT MPI compared with ${ }^{18} \mathrm{~F}$-flurpiridaz PET MPI in patients with smaller LVs?

PERTINENT FINDINGS: Whereas the performance of SPECT MPI decreases significantly in smaller compared with larger LVs, it is similar in PET MPI regardless of LV size. Unlike SPECT MPI, there is no degradation of sensitivity in smaller LVs with PET MPI.

IMPLICATIONS FOR PATIENT CARE: PET MPI is not affected by LV size, is superior to SPECT MPI in patients with smaller LVs, and should be the preferred method for the detection and evaluation of $C A D$ in such patients.

\section{REFERENCES}

1. Hansen CL, Crabbe D, Rubin S. Lower diagnostic accuracy of thallium-201 SPECT myocardial perfusion imaging in women: an effect of smaller chamber size. J Am Coll Cardiol. 1996;28:1214-1219.

2. Salton CJ, Chuang ML, O'Donnell CJ, et al. Gender differences and normal left ventricular anatomy in an adult population free of hypertension: a cardiovascular magnetic resonance study of the Framingham Heart Study Offspring Cohort. $J$ Am Coll Cardiol. 2002;39:1055-1060.

3. Nakajima K, Okuda K, Kawano M, et al. The importance of population-specific normal database for quantification of myocardial ischemia: comparison between Japanese 360 and 180-degree databases and a US database. J Nucl Cardiol. 2009;16:422-430.

4. Li D, Li D, Feng J, Yuan D, Cao K, Chen J. Quantification of myocardial perfusion SPECT studies in Chinese population with Western normal databases. $J$ Nucl Cardiol. 2010;17:486-493.

5. Echocardiographic Normal Ranges Meta-Analysis of the Left Heart Collaboration. Ethnic-specific normative reference values for echocardiographic LA and LV size, LV mass, and systolic function: the EchoNoRMAL study. JACC Cardiovasc Imaging. 2015;8:656-665.

6. Maddahi J, Packard RR. PET should replace SPECT in cardiac imaging for diagnosis and risk assessment of patients with known or suspected CAD: Pro. $J$ Nucl Cardiol. 2017;24:1955-1959.

7. Vallejo E, Dione DP, Bruni WL, et al. Reproducibility and accuracy of gated SPECT for determination of left ventricular volumes and ejection fraction: experimental validation using MRI. J Nucl Med. 2000;41:874-882.

8. Abidov A, Germano G, Hachamovitch R, Slomka P, Berman DS. Gated SPECT in assessment of regional and global left ventricular function: an update. $\mathrm{J} \mathrm{Nucl}$ Cardiol. 2013;20:1118-1143.

9. Maddahi J, Packard RR. Cardiac PET perfusion tracers: current status and future directions. Semin Nucl Med. 2014;44:333-343.

10. Dorbala S, Di Carli MF. Cardiac PET perfusion: prognosis, risk stratification, and clinical management. Semin Nucl Med. 2014;44:344-357.

11. Bateman TM, Dilsizian V, Beanlands RS, DePuey EG, Heller GV, Wolinsky DA. American Society of Nuclear Cardiology and Society of Nuclear Medicine and Molecular Imaging joint position statement on the clinical indications for myocardial perfusion PET. J Nucl Med. 2016;57:1654-1656.

12. Schindler TH, Quercioli A, Valenta I, Ambrosio G, Wahl RL, Dilsizian V. Quantitative assessment of myocardial blood flow: clinical and research applications. Semin Nucl Med. 2014;44:274-293.

13. Berman DS, Maddahi J, Tamarappoo BK, et al. Phase II safety and clinical comparison with single-photon emission computed tomography myocardial perfusion imaging for detection of coronary artery disease: flurpiridaz $\mathrm{F} 18$ positron emission tomography. J Am Coll Cardiol. 2013;61:469-477.

14. Maddahi J, Lazewatsky J, Udelson JE, et al. Phase III clinical trial of fluorine-18 flurpiridaz positron emission tomography for evaluation of coronary artery disease. J Am Coll Cardiol. 2020;76:391-401.

15. Parker MW, Iskandar A, Limone B, et al. Diagnostic accuracy of cardiac positron emission tomography versus single photon emission computed tomography for coronary artery disease: a bivariate meta-analysis. Circ Cardiovasc Imaging. 2012;5:700-707.

16. Mc Ardle BA, Dowsley TF, deKemp RA, Wells GA, Beanlands RS. Does rubidium-82 PET have superior accuracy to SPECT perfusion imaging for the diagnosis of obstructive coronary disease? A systematic review and meta-analysis. J Am Coll Cardiol. 2012;60:1828-1837.

17. Einstein AJ, Berman DS, Min JK, et al. Patient-centered imaging shared decision making for cardiac imaging procedures with exposure to ionizing radiation. $J \mathrm{Am}$ Coll Cardiol. 2014;63:1480-1489.

18. Fihn SD, Gardin JM, Abrams J, et al. 2012 ACCF/AHA/ACP/AATS/PCNA/ SCAI/STS guideline for the diagnosis and management of patients with stable ischemic heart disease. Circulation. 2012;126:e354-e471.

19. Wolk MJ, Bailey SR, Doherty JU, et al. ACCF/AHA/ASE/ASNC/HFSA/HRS/ SCAI/SCCT/SCMR/STS 2013 multimodality appropriate use criteria for the detection and risk assessment of stable ischemic heart disease. J Am Coll Cardiol. 2014;63:380-406.

20. Kay J, Dorbala S, Goyal A, et al. Influence of sex on risk stratification with stress myocardial perfusion $\mathrm{Rb}-82$ positron emission tomography: results from the PET (positron emission tomography) prognosis multicenter registry. J Am Coll Cardiol. 2013;62:1866-1876.

21. Chow BJ, Dorbala S, Di Carli MF, et al. Prognostic value of PET myocardial perfusion imaging in obese patients. JACC Cardiovasc Imaging. 2014;7:278-287.

22. Huisman MC, Higuchi T, Reder S, et al. Initial characterization of an ${ }^{18} \mathrm{~F}$-labeled myocardial perfusion tracer. $J$ Nucl Med. 2008;49:630-636.

23. Dorbala S, Vangala D, Sampson U, Limaye A, Kwong R, Di Carli MF. Value of vasodilator left ventricular ejection fraction reserve in evaluating the magnitude of myocardium at risk and the extent of angiographic coronary artery disease: ${ }^{82} \mathrm{Rb}$ PET/CT study. J Nucl Med. 2007;48:349-358.

24. Packard RR, Huang SC, Dahlbom M, Czernin J, Maddahi J. Absolute quantitation of myocardial blood flow in human subjects with or without myocardial ischemia using dynamic flurpiridaz F 18 PET. J Nucl Med. 2014;55:1438-1444.

25. Moody JB, Poitrasson-Rivière A, Hagio T, et al. Added value of myocardial blood flow using ${ }^{18} \mathrm{~F}$-flurpiridaz PET to diagnose coronary artery disease. J Nucl Cardiol. January 30, 2020 [Epub ahead of print].

26. Flotats A, Bravo PE, Fukushima K, Chaudhry MA, Merrill J, Bengel FM. ${ }^{82} \mathrm{Rb}$ PET myocardial perfusion imaging is superior to ${ }^{99 \mathrm{~m}}$ Tc-labelled agent SPECT in patients with known or suspected coronary artery disease. Eur J Nucl Med Mol Imaging. 2012;39:1233-1239.

27. Bateman TM, Heller GV, McGhie AI, et al. Diagnostic accuracy of rest/stress ECG-gated Rb-82 myocardial perfusion PET: comparison with ECG-gated Tc-99m sestamibi SPECT. J Nucl Cardiol. 2006;13:24-33.

28. Tonino PA, Fearon WF, De Bruyne B, et al. Angiographic versus functional severity of coronary artery stenoses in the FAME study: fractional flow reserve versus angiography in multivessel evaluation. J Am Coll Cardiol. 2010;55:2816-2821.

29. Mielniczuk LM, Toth GG, Xie JX, De Bruyne B, Shaw LJ, Beanlands RS. Can functional testing for ischemia and viability guide revascularization? JACC Cardiovasc Imaging. 2017;10:354-364.

30. Maddahi J. Properties of an ideal PET perfusion tracer: new PET tracer cases and data. J Nucl Cardiol. 2012;19(supp1 1):S30-S37.

31. Maddahi J, Czernin J, Lazewatsky J, et al. Phase I, first-in-human study of BMS747158, a novel F-18-labeled tracer for myocardial perfusion PET: dosimetry, biodistribution, safety, and imaging characteristics after a single injection at rest. J Nucl Med. 2011;52:1490-1498.

32. Maddahi J, Bengel F, Czernin J, et al. Dosimetry, biodistribution, and safety of flurpiridaz F18 in healthy subjects undergoing rest and exercise or pharmacological stress PET myocardial perfusion imaging. J Nucl Cardiol. 2019;26:2018-2030.

33. Packard RR, Cooke CD, Van Train KF, et al. Development, diagnostic performance, and interobserver agreement of a ${ }^{18} \mathrm{~F}$-flurpiridaz PET automated perfusion quantitation system. J Nucl Cardiol. September 7, 2020 [Epub ahead of print].

34. Bravo PE, Chien D, Javadi M, Merrill J, Bengel FM. Reference ranges for LVEF and LV volumes from electrocardiographically gated ${ }^{82} \mathrm{Rb}$ cardiac PET/CT using commercially available software. J Nucl Med. 2010;51:898-905.

35. DuBois D, DuBois EF. A formula to estimate the approximate surface area if height and weight be known. Arch Intern Med. 1916;17:863-871. 